Case Report

\title{
Atypical Presentation of Cat-Scratch Disease in an Immunocompetent Child with Serological and Pathological Evidence
}

\author{
Serkan Atıcı, ${ }^{1}$ Eda Kepenekli Kadayıfcı, ${ }^{1}$ Ayşe Karaaslan, ${ }^{1}$ Muhammed Hasan Toper, \\ Cigdem Ataizi Celikel, ${ }^{2}$ Ahmet Soysal, ${ }^{1,3}$ and Mustafa Bakur ${ }^{1}$ \\ ${ }^{1}$ Department of Pediatrics and Division of Pediatric Infectious Diseases, Marmara University Medical Faculty, \\ Pendik Training and Research Hospital, Fevzi Cakmak Mah. Mimar Sinan Cad., Ust Kaynarca, Pendik, 34899 Istanbul, Turkey \\ ${ }^{2}$ Department of Pathology, Marmara University Medical Faculty, Pendik Training and Research Hospital, \\ Fevzi Cakmak Mah. Mimar Sinan Cad., Ust Kaynarca, Pendik, 34899 Istanbul, Turkey \\ ${ }^{3}$ Marmara University Medical Faculty, Pendik Training and Research Hospital, Fevzi Cakmak Mah. Mimar Sinan Cad. \\ Ust Kaynarca, Pendik, 34899 Istanbul, Turkey \\ Correspondence should be addressed to Ahmet Soysal; ahsoysal@yahoo.com
}

Received 5 July 2014; Accepted 7 December 2014; Published 28 December 2014

Academic Editor: Bibhuti Das

Copyright (C) 2014 Serkan Atıc1 et al. This is an open access article distributed under the Creative Commons Attribution License, which permits unrestricted use, distribution, and reproduction in any medium, provided the original work is properly cited.

Typical cat-scratch disease (CSD) is characterized by local lymphadenopathy following the scratch or bite from a cat or kitten. An atypical presentation which includes liver and/or spleen lesions is rarely reported in an immunocompetent child. Systemic CSD may mimic more serious disorders like malignancy or tuberculosis. Although a diagnosis is difficult to establish in systemic CSD, an early diagnosis and an appropriate treatment are important to prevent complications. Bartonella henselae is difficult to culture, and culture is not routinely recommended. Clinical, serological, radiological, and pathological findings are used for the diagnosis of CSD. Herein we present a case of systemic CSD presenting with hepatic mass in an immunocompetent child. The differential diagnosis is made by serological and pathological evidence. He was successfully treated with gentamicin $(7.5 \mathrm{mg} / \mathrm{kg})$ and rifampin $(15 \mathrm{mg} / \mathrm{kg})$ for six weeks.

\section{Introduction}

Cat-scratch disease (CSD) is an infectious disease caused by the Bartonella henselae. It is a small, curved, aerobic, slowgrowing, fastidious, gram-negative, intracellular Bacillus that causes granulomatous inflammation of the tissue and it can be painted with silver stain [1].

CSD is usually associated with a previous history of exposure to cats or kittens. Although a history of scratch, bite, or licking from a cat or kitten is important, it is not necessary for the diagnosis. Typical CSD is characterized by local lymphadenopathy with or without skin rash and is usually self-limited. Systemic CSD may present in a more disseminated form which usually occurs in immunocompromised children. Atypical presentations which include hepatic and/or splenic lesions, osteomyelitis, discitis, granulomatous conjunctivitis, endocarditis, myocarditis, neuroretinitis, and encephalomeningitis may mimic more serious disorders such as malignancy and could be an important differential diagnostic problem $[1,2]$. Isolated hepatic lesions of CSD are a rare clinical condition especially in an immunocompetent child.

We report the case of an immunocompetent child diagnosed as systemic CSD with serological and pathological evidence. He had multiple hepatic lesions. He was successfully treated with intravenous gentamicin and oral rifampin. The aim of this study was to report the management of hepatic CSD in an immunocompetent child.

\section{Case Report}

A 12-year-old boy was admitted to another hospital with 7day history of fever, abdominal pain, headache, and weight loss. Empirical antibiotics therapy including ceftriaxone and 


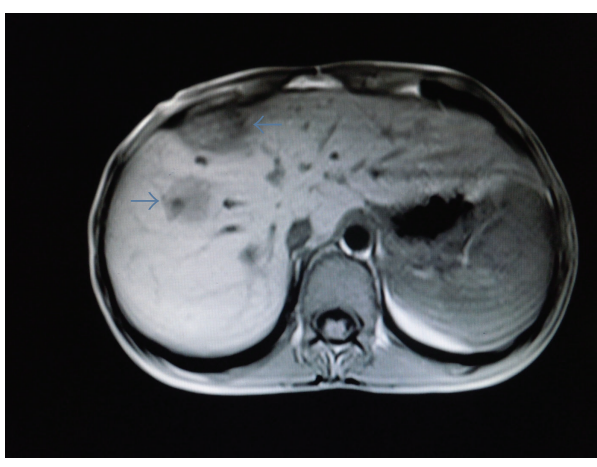

(a)

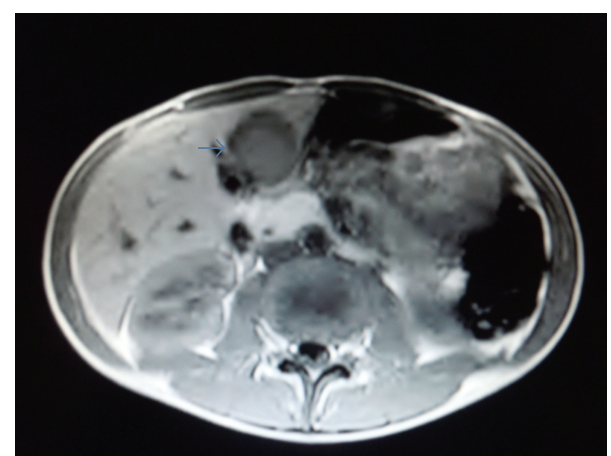

(b)

FIGURE 1: T1 (a) and T2 (b) weighted magnetic resonance imaging shows multiple lesions in the liver.

clindamycin had been administered. After the abdominal ultrasound demonstrated multiple hypoechoic liver lesions, he was transferred to our department on the eleventh day of hospitalization. Fever and abdominal pain continued and he had lost 8 kilograms. He had a history of playing with a kitten. Physical examination revealed bilateral inguinal lymphadenopathy. There was not any scratch or papule on his skin. His past medical history was not consistent with any primary or secondary immunodeficiency or any other underlying disease. Laboratory findings included the following: white blood cell count, $10400 / \mathrm{mm}^{3}$; hemoglobin, $11.9 \mathrm{~g} / \mathrm{dL}$; platelets, $385000 / \mathrm{mm}^{3}$; C-reactive protein (CRP), $10.1 \mathrm{mg} / \mathrm{dL}$; erythrocyte sedimentation rate (ESR), $57 \mathrm{~mm}$ in $1 \mathrm{~h}$; aspartate aminotransferase (AST), $168 \mathrm{U} / \mathrm{L}$; alanine aminotransferase (ALT), $67 \mathrm{U} / \mathrm{L}$. Blood and urine cultures were negative. Serology of human immunodeficiency virus was also negative. Abdominal magnetic resonance imaging (MRI) showed multiple hepatic lesions (Figure 1). Cranial and thoracal imaging revealed no distinct abnormality. Serological analyses by indirect fluorescent antibody (IFA) method detected the presence of immunoglobulin (Ig) G and IgM antibodies to Bartonella henselae positive with a titer of $1: 320$ and $1: 100$, respectively. He was assessed to the pediatric immunology department with suspected immunodeficiency. Lymphocyte subset analysis, dihydrorhodamine 123 flow cytometry, and the serum immunoglobulins levels were normal. Ultrasound guided liver biopsy was performed. Histopathological analyses of the lesions showed granulomas surrounded with palisade histiocytes and non calcified necrosis with hematoxylin and eosin (H\&E) staining (Figure 2(a)). Acid-fast Bacilli were not detected with EhrlichZiehl-Neelsen (EZN) staining (Figure 2(b)). Warthin-Starry silver stain was positive which was compatible with CSD (Figure 2(c)).

He was treated with intravenous gentamicin $(7.5 \mathrm{mg} / \mathrm{kg})$ and oral rifampin $(15 \mathrm{mg} / \mathrm{kg})$ for six weeks. His symptoms were resolved and abnormal laboratory findings including elevated CRP, ESR, and liver transaminases were normalized after antibiotic therapy. Contrast enhanced abdominal MRI was repeated on 30th day of antibiotic therapy and showed that hepatic lesions had regressed.

\section{Discussion}

Bartonella henselae is the etiologic agent of cat-scratch disease and cats are the major reservoir for the bacteria. A study in our country determined Bartonella henselae IgG antibody seroprevalence was $18.8 \%$ in cats. Seropositivity was observed as $27.5 \%$ for stray cats. The seropositivity was $14.3 \%$ and $11.4 \%$ in outdoor and indoor domestic cats in Turkey, respectively [3]. History of scratch or bite from a cat or kitten is helpful for the diagnosis. Our patient had a history of playing with a kitten.

Although most patients with CSD typically presented with fever and lymphadenopathy, atypical clinical manifestations may be seen.Atypical clinical presentations of CSD include a broad spectrum of clinical syndromes ranging from prolonged fever of unknown origin to hepatosplenic, ocular, and neurological manifestations $[4,5]$. Hepatosplenic disease is an unusual clinic presentation occurring in only $0.3 \%$ to $0.7 \%$ of patients, mostly in children [6]. Hepatic involvement is an uncommon clinical presentation in immunocompetent children. Patients with hepatic lesions present episodic abdominal pain with prolonged fever. Although liver enzymes are usually normal, erythrocyte sedimentation rate is often increased.

Abdominal imaging is an important diagnostic step in patients with suspected hepatosplenic CSD [4]. Ultrasonography (US), computed tomography (CT), or MRI may show multiple and variable size and shape lesions in the liver or spleen. Lesions are usually hypoechoic on US and hypoattenuated on CT. Multiple hepatic lesions may be due to lymphoma, histoplasmosis, granulomatous processes, and metastatic disease $[7,8]$.

Bartonella henselae is difficult to culture, and culture is not routinely recommended. Clinical, serological, radiological, and pathological findings are used for the diagnosis of CSD. In history, the exposure to animals, especially cats, is important medical information to suspect from CSD [9]. Serological testing for Bartonella henselae antibodies is the most cost-effective diagnostic modality with IFA to be most frequently used $[4,10]$. Granulomatous inflammation is not specific to CSD, so other granulomatous diseases such as tuberculosis should be considered. Although tuberculosis 


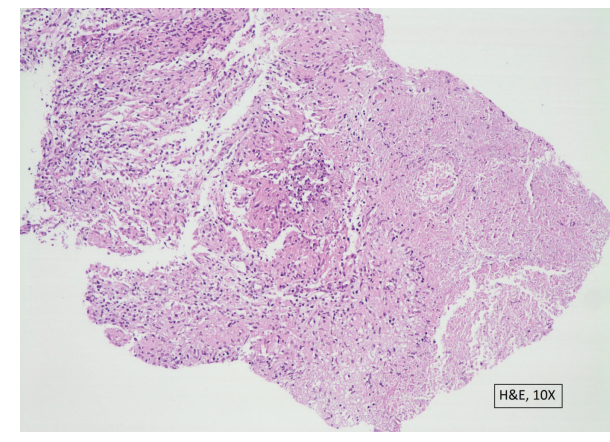

(a)

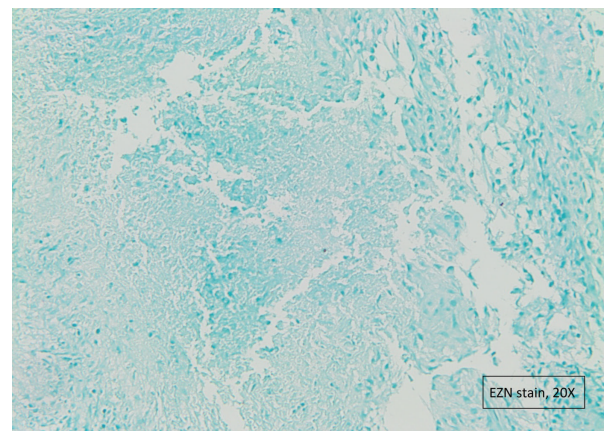

(b)

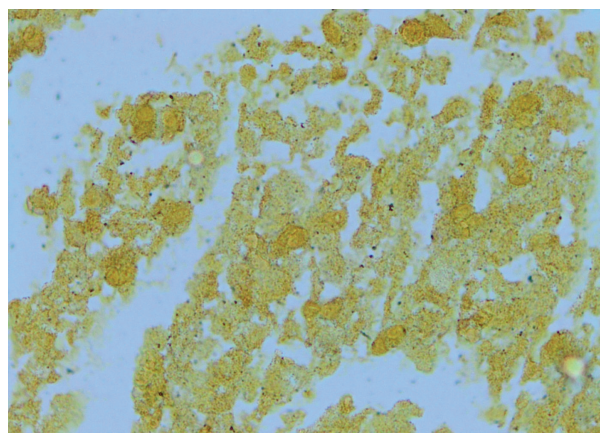

(c)

FIGURE 2: (a) The liver lesion is characterized by multiple granulomas hematoxylin and eosin $\times 10$, (b) acid-fast Bacilli was not detected with EZN staining $\times 10$, and (c) Bartonella henselae was demonstrated by Warthin-Starry silver stain $\times 10$.

is still common in Turkey, isolated hepatic lesions are unusual. We have investigated for tuberculosis and ruled out histopathological findings and laboratory tests including IFN-gamma releasing assay (QuantiFERON-TB), EZN staining, and mycobacterial culture of gastric lavage fluids. Acidfast Bacillus was not detected on liver biopsy with EhrlichZiehl-Neelsen staining but Bartonella henselae has been demonstrated as short rods by using Warthin-Starry silver stain (Figure 2(c)). Previous history of contact with kitten, the positive serology for Bartonella henselae, and radiological and pathological findings were used for the diagnosis of our patient.

Systemic CSD has a high morbidity rate in immunocompromised children. There is no consensus about the type of antimicrobial drug and the duration of the therapy for the diagnosis of systemic CSD in an immunocompetent child [11]. Rifampin, trimethoprim-sulfamethoxazole, gentamicin, macrolides, extended spectrum cephalosporins, and ciprofloxacin all have in vitro activity against Bartonella henselae [11, 12]. Arısoy et al. published a large series including 19 children with hepatosplenic cat-scratch disease and all patients were treated with one or more antibiotics including gentamicin $(7.5 \mathrm{mg} / \mathrm{kg})$, rifampin $(15-20 \mathrm{mg} / \mathrm{kg})$, and trimethoprim-sulfamethoxazole $(10-12 \mathrm{mg} / \mathrm{kg})$ for 10 to 21 days. Thirteen patients were treated with rifampin alone and 3 patients were treated with rifampin plus gentamicin or trimethoprim-sulfamethoxazole. Rifampin was proposed in the antimicrobial treatment of hepatosplenic CSD in that study [12]. We used a rifampin plus gentamicin combination therapy in our case for 6 weeks. His symptoms were resolved and the hepatic lesions regressed. A relapse was not determined during the follow-up.

\section{Conflict of Interests}

The authors declare that they have no conflict of interests.

\section{References}

[1] S. A. Klotz, V. Ianas, and S. P. Elliott, "Cat-scratch disease," American Family Physician, vol. 83, no. 2, pp. 152-155, 2011.

[2] M. Kojić, D. Mikić, D. Nožić, and L. Zolotarevski, "Atypical form of cat scratch disease in immunocompetent patient," Vojnosanitetski Pregled, vol. 70, no. 1, pp. 72-76, 2013.

[3] B. Celebi, S. Kilic, N. Aydin, G. Tarhan, A. Carhan, and C. Babur, "Investigation of Bartonella henselae in cats in Ankara, Turkey," Zoonoses and Public Health, vol. 56, no. 4, pp. 169-175, 2009.

[4] T. A. Florin, T. E. Zaoutis, and L. B. Zaoutis, "Beyond cat scratch disease: widening spectrum of Bartonella henselae infection," Pediatrics, vol. 121, no. 5, pp. e1413-e1425, 2008.

[5] E. Eskow, R.-V. S. Rao, and E. Mordechai, "Concurrent infection of the central nervous system by Borrelia burgdorferi and Bartonella henselae: evidence for a novel tick-borne disease complex," Archives of Neurology, vol. 58, no. 9, pp. 1357-1363, 2001.

[6] G. M. Marsilia, A. la Mura, R. Galdiero, E. Galdiero, G. Aloj, and A. Ragozzino, "Isolated hepatic involvement of cat scratch 
disease in immunocompetent adults: enhanced magnetic resonance imaging, pathological findings, and molecular analysistwo cases," International Journal of Surgical Pathology, vol. 14, no. 4, pp. 349-354, 2006.

[7] A. Rohr, M. R. Saettele, S. A. Patel, C. A. Lawrence, and L. H. Lowe, "Spectrum of radiological manifestations of paediatric cat-scratch disease," Pediatric Radiology, vol. 42, no. 11, pp. 13801384, 2012.

[8] O. Danon, M. Duval-Arnould, Z. Osman et al., "Hepatic and splenic involvement in cat-scratch disease: imaging features," Abdominal Imaging, vol. 25, no. 2, pp. 182-183, 2000.

[9] T. Koga, J. Taguchi, M. Suzuki et al., "Cat scratch disease presenting with a retroperitoneal abscess in a patient without animal contacts," Journal of Infection and Chemotherapy, vol. 15, no. 6, pp. 414-416, 2009.

[10] D. Anyfantakis, M. Kastanakis, A. Papadomichelakis, G. Petrakis, and E. Bobolakis, "Cat-scratch disease presenting as a solitary splenic abscess in an immunocompetent adult: case report and literature review," Infezioni in Medicina, vol. 21, no. 2, pp. 130-133, 2013.

[11] C. Scolfaro, G. G. K. Leunga, S. Bezzio et al., "Prolonged follow up of seven patients affected by hepatosplenic granulomata due to cat-scratch disease," European Journal of Pediatrics, vol. 167, no. 4, pp. 471-473, 2008.

[12] E. S. Arisoy, A. G. Correa, M. L. Wagner, and S. L. Kaplan, "Hepatosplenic cat-scratch disease in children: selected clinical features and treatment," Clinical Infectious Diseases, vol. 28, no. 4, pp. 778-784, 1999. 


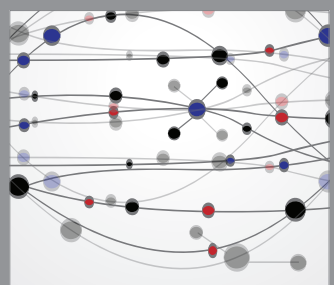

The Scientific World Journal
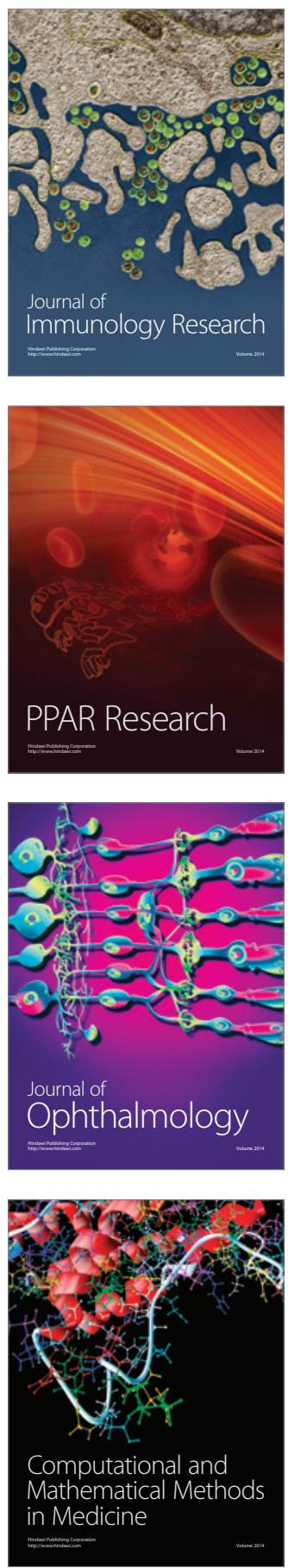

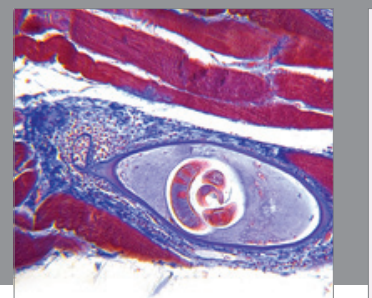

Gastroenterology

Research and Practice
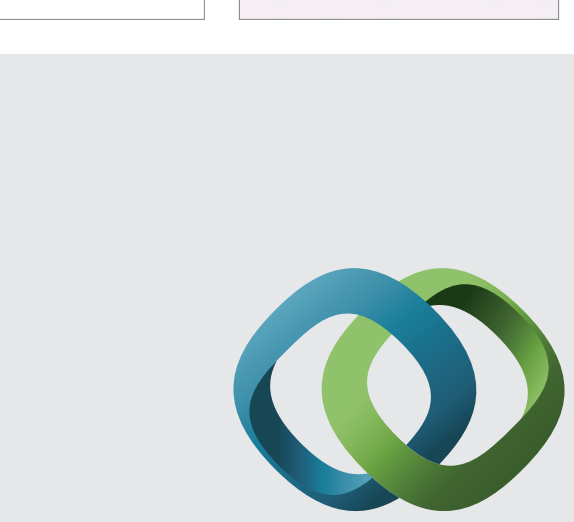

\section{Hindawi}

Submit your manuscripts at

http://www.hindawi.com
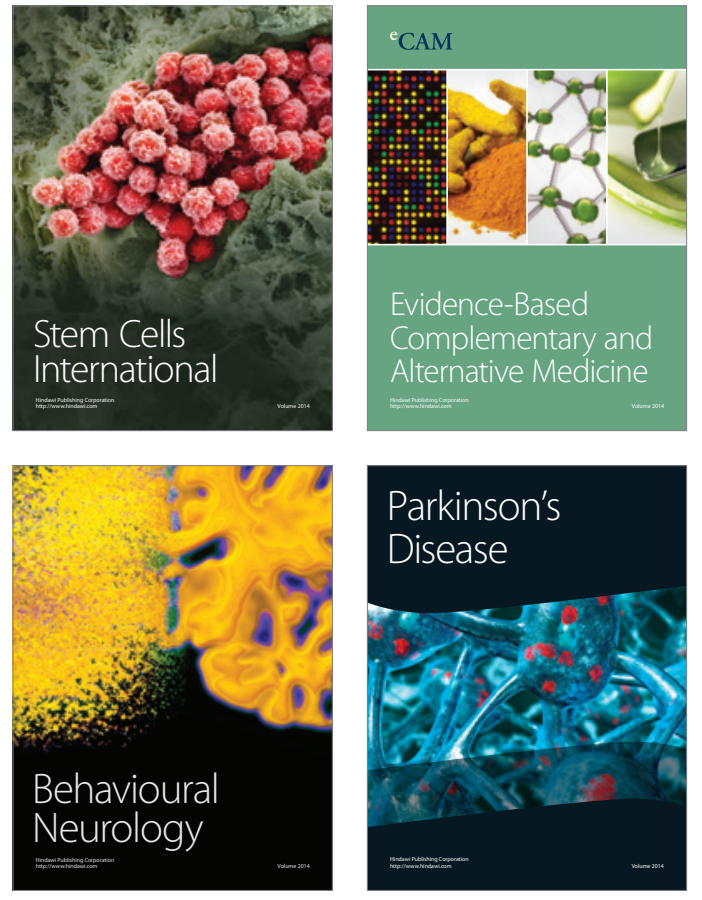
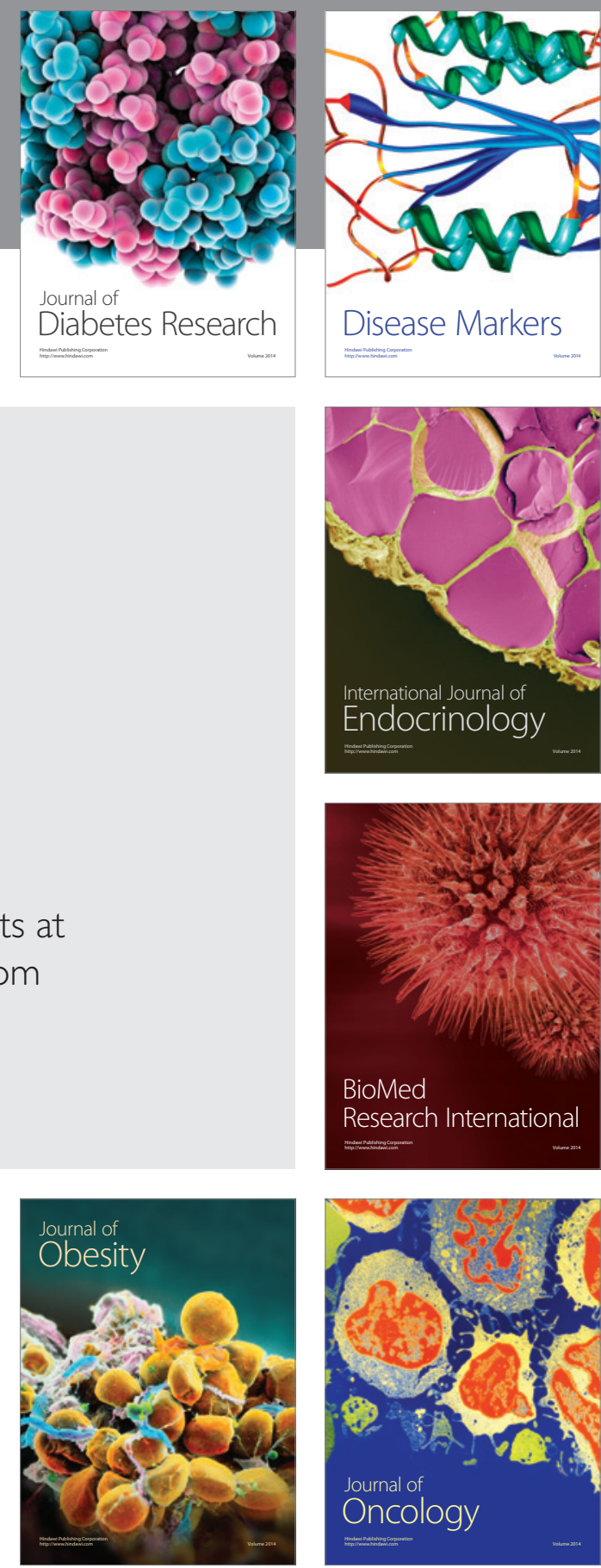

Disease Markers
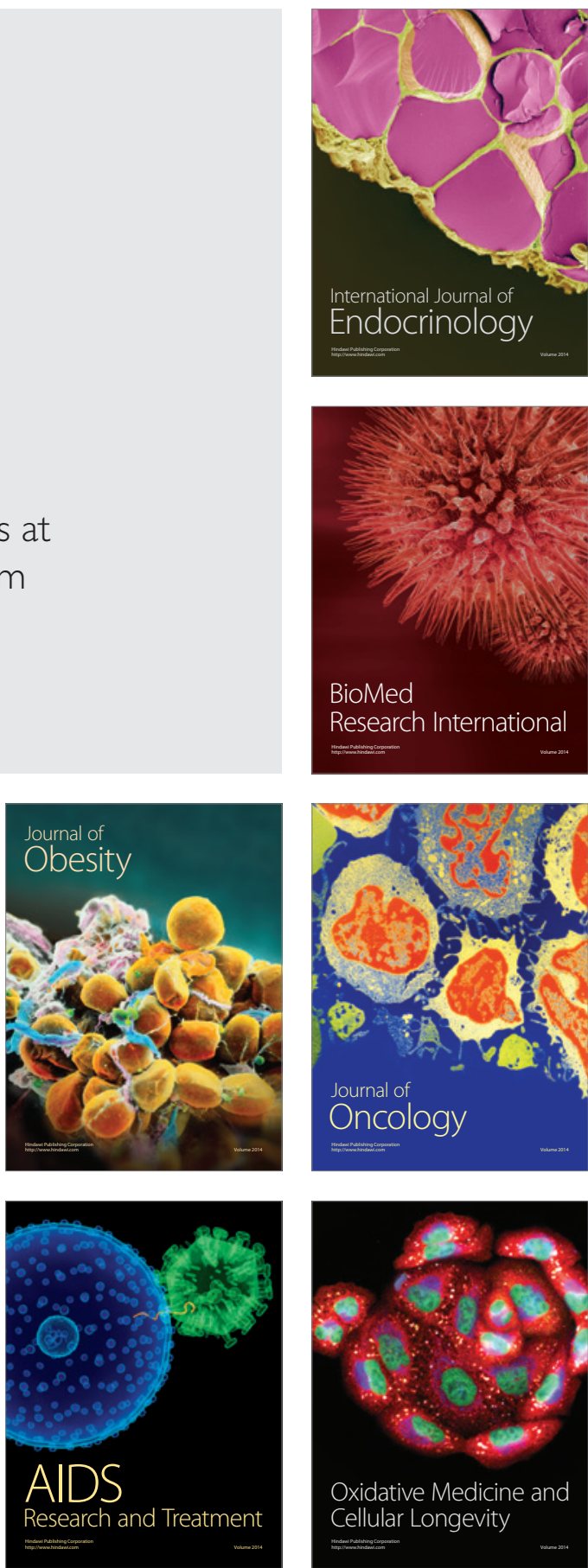